\title{
Wie kann die Rate von BPD bei sehr kleinen Frühgeborenen gesenkt werden?
}

\section{Bereits in der Vergangenheit wurden verschiedene Therapie- konzepte zur Vermeidung einer bronchopulmonale Dysplasie getestet, insbesondere unterschiedliche Kortisonpräparate (inha- latives Budesonid, Dexamethason, intravenöses Hydrocor-tison). In einer französischen Studie wurde nun der frühe Einsatz von „low dose“-Hydrocortison i.v. untersucht.}

") Die bronchopulmonale Dysplasie (BPD), auch chronische Lungenerkrankung der Frühgeborenen genannt, ist eine der wesentlichen Komplikationen bei sehr kleinen und unreifen Kindern vor der 28. Schwangerschaftswoche. Eine groß angelegte, placebokontrollierte, doppelblinde Studie wurde nun in der Zeitschrift Lancet vorgestellt: Über einen Zeitraum von sechs Jahren konnten in insgesamt 21 französischen Neugeborenenintensivstation mehr als 1.000 Frühgeborene für die Studie untersucht und letztendlich 521 einbezogen werden: 255 Kinder wurden der Hydrocortison-Gruppe und 266 Kinder der Placebogruppe zugewiesen.
$153(60 \%)$ der Verumgruppe überlebten ohne BPD verglichen mit 136 (51\%) der Placebogruppe (Odds Ratio: 1,48; 95\%-Konfidenzintervall 1,02-2,16; $\mathrm{p}=$ 0,04). Außerdem konnten die Kinder unter Hydrocortison früher extubiert werden. Die Rate an Sepsis war nicht signifikant unterschiedlich in den beiden Gruppen, in einer Subgruppe der Hydrocortison-Gruppe mit Kindern aus 24-25 Schwangerschaftswochen war der Prozentsatz jedoch erhöht.

Bezüglich anderer möglicher Komplikationen, vor allem gastrointestinaler Perforationen, konnten in beiden Gruppen keine signifikanten Unterschiede gefunden werden. Auch die seltene, aber gefürchtete Komplikation

\section{Kommentar}

Dies ist eine der größten randomisierten und kontrollierten Studien mit einer sehr frühen (am 2. Lebenstag) und dabei relativ niedrigen Gabe von Hydrocortison. Und sie konnte zeigen, dass die Kortisontherapie die Rate der Kinder signifikant verbessert, die später ohne zusätzliche Sauerstoffgabe und damit ohne BPD das Reifealter erreichen. Darüber hinaus zeigt sie auch die recht gute Sicherheitslage dieser Kurzzeit-Intervention, bei der insbesondere keine bedrohliche Zunahme von gastrointestinalen Perforationen, Sepsis oder Kardiomyopathien aufgetreten ist. Neurologische Spätfolgen konnten mit dieser Studie mit Ergebnissen aus den Jahren 2008-2014 noch nicht ausgeschlossen werden, jedoch war bezüglich Hirnblutungen und anderer zerebralen Komplikationen keine Zunahme erkennbar.

Das Auftreten erhöhter Sepsiszahlen in einer - zugegebenermaßen kleinen Subgruppe sollte jedoch Anlass zur Vorsicht und besonderen Aufmerksamkeit gerade bei sehr unreifen Kindern unter Hydrocortison geben, ohne deswegen auf die positiven Effekte des Medikaments zu verzichten. Auch erwies sich in der Studie die Gabe von Hydrocortison gegenüber Placebo günstiger bezüglich einer erniedrigten Rate von Ligaturen eines persistierenden Ductus Botalli. Vergleichbare Studien mit Dexamethason [Pera A et al. J Perinatol 2002;22:20408; Paech C et al. J Perinatol 2014;34:244-46], inhalativem Budesonid [Bassler D et al. N Engl J Med 2015;373:1497-506] und eine Cochrane-Übersicht über postnatale Kortikosteroide [Doyle LW et al. Cochrane Database Syst Rev 2014;5:CD001146] zeigen, dass die hier vorgeschlagene frühe und relativ kurze Intervention mit Hydrocortison gegenüber anderen Präparaten effektiver und vor allem auch sicherer ist. Als hauptsächlicher Wirkmechanismus wird eine Stabilisierung beziehungsweise Anhebung des Blutdrucks und die Reduktion inflammatorischer Prozesse angenommen. So zeigte auch eine spezielle Analyse, dass ein Benefit von Hydrocortison vor allem auch bei Kindern mit mütterlicher Chorioamnionitis bestand. der hypertrophen Kardiomyopathie kam nur bei einem Kind der Hydrocortison- und bei einem Kind der Placebogruppe vor und muss damit auch ätiologisch wahrscheinlich anders zugeordnet werden. Drei Kinder der Hydrocortison-Gruppe, aber demgegenüber acht Kinder der Placebogruppe, wiesen eine Candida-Sepsis auf.

$\mathrm{Zu}$ weiteren, ernsten Komplikationen (v.a. Hirnblutungen, periventrikuläre Leukomalazie, nekrotisierende Enterokolitis) konnte festgestellt werden: 70 Kinder der 24.-25. Schwangerschaftswoche (84\%) unter Hydrocortison hatten mindestens eine dieser Komplikationen, verglichen mit 80 (89\%) der 90 Kinder der Placebogruppe. Bei den Kindern aus der 26.-27. Schwangerschaftswoche betrugen die Zahlen 53\% beziehungsweise 59\%.

Die Autoren schlussfolgern, dass die frühe tägliche i.v.-Gabe von $2 \times 0,5 \mathrm{mg} /$ kg KG Hydrocortison über sieben Tage, gefolgt von 1 x 0,5 mg/kg KG Hydrocortison über drei Tage ohne schwerwiegendere Komplikationen die Wahrscheinlichkeit einer BPD gegenüber Placebo signifikant senkt. BPD war hier - wie meist international üblich als Sauerstoffbedarf jenseits der 36 . postmenstruellen Lebenswoche definiert.

Quelle:

Baud O et al. Effect of early low-dose hydrocortisone on survival without bronchopulmonary dysplasia in extremely preterm infants (PREMILOC): a doubleblind, placebo-controlled, multicentre, randomised trial. Lancet 2016;387: 1827-36

Autorenkontakt:

Referiert und kommentiert von Dr. med. Ulrich Mutschler. Der Hamburger Kinderarzt ist auch Chefredakteur der Zeitschrift „Pädiatrie“.

E-Mail: u.mutschler@gmx.net 